\title{
Analysis of the Electrical Heart Field
}

\author{
O. KITTNAR ${ }^{1}$, M. MLČEK ${ }^{1}$ \\ ${ }^{1}$ Institute of Physiology, First Faculty of Medicine, Charles University in Prague, Prague, Czech \\ Republic
}

Received February 3, 2010

Accepted March 26, 2010

\section{Summary}

There are three basic procedures used for an assessment of the electrical heart field from the body surface: standard electrocardiography, vectorcardiography, and body surface potential mapping (BSPM). BSPM has two major advantages over both other methods: 1) it allows exploring the entire chest surface, thus providing all the information on the cardiac electric field available at the body surface; 2 ) it is more sensitive in detecting local electrical events, such as local conduction disturbances or regional heterogeneities of ventricular recovery. Nevertheless the results obtained using BSPM procedure cannot answer all questions about real causality of detected changes of the electrical heart field. We tried therefore to use mathematical model of the electrical field in order to answer these questions. A simple and anatomical forward calculation model was used to test the hypothesis whether the altered position of the heart could explain heterogeneity of repolarization at late stages of pregnancy in humans. The hypothesis was declined. Further findings included: A. Repolarization duration (represented by QT interval) in healthy subjects are distributed regularly and predictably on the body surface carrying no information about local pathology. B. At any systemic analysis of ventricular repolarization, it is vital to consider the regions where any electrode systems record low amplitudes due to methodological, not pathological reasons. C. Anatomical (heterogeneous) model did not yield superior results over simple (homogenous) ones possibly since none reflected the specific torso geometry of individual patients.

\section{Key words}

Electrocardiography - Body surface potential mapping • Mathematical modeling

\section{Corresponding author}

O. Kittnar, Institute of Physiology, Charles University in Prague, First Faculty of Medicine, Albertov 5, 12800 Prague 2, Czech Republic. E-mail: okittnar@lf1.cuni.cz

\section{Introduction}

There are several procedures suggested to assess electrical heart field from the body surface. Nevertheless, all of them are facing the same problems (Fig. 1):

- the source of the electrical field is located asymmetrically in a thorax,

- different tissues between the source and the body surface have also different impedance,

- the source is a complex figure with very complicated geometry and its electrical field is a result of space summation of electrical fields of individual myocardial cells.

Thus it can be concluded that the surface electrical heart field "reflects complex and interrelated aspects of cardiac electrophysiology, cardiac geometry, torso shape, tissue impedance, and biological signal processing" (Higham and Campbell 1994).

In fact there are three basic procedures used for an assessment of the electrical heart field from the body surface: standard electrocardiography, vectorcardiography, and body surface potential mapping (BSPM). Twelve-lead electrocardiography is the oldest and most frequently used method, it is well defined and has relevant clinical applications that are accepted all over the world. On the other hand it does not reflect the torso geometry; the lead system is based just on the empiric approach of fathers of electrocardiography. 
Vectorcardiography represents orthogonal lead system. Thanks to this fact it corresponds better to the complex geometry of the electrical heart field. But it does not allow assessing arrhythmias and it never became a standardly used clinical method. The BSPM uses multilead system (from 24 to more than 200 leads) and thus provides detailed spatial and temporal information on the electric activity of the heart from the body-surface measurements. In fact both standard electrocardiography and vectorcardiography are used for the same purpose; however, they sample the body surface potentials only at a limited number of sites. On the other hand the BSPM is very complicated for clinical interpretation and thus it has never become a standardly used clinical method.

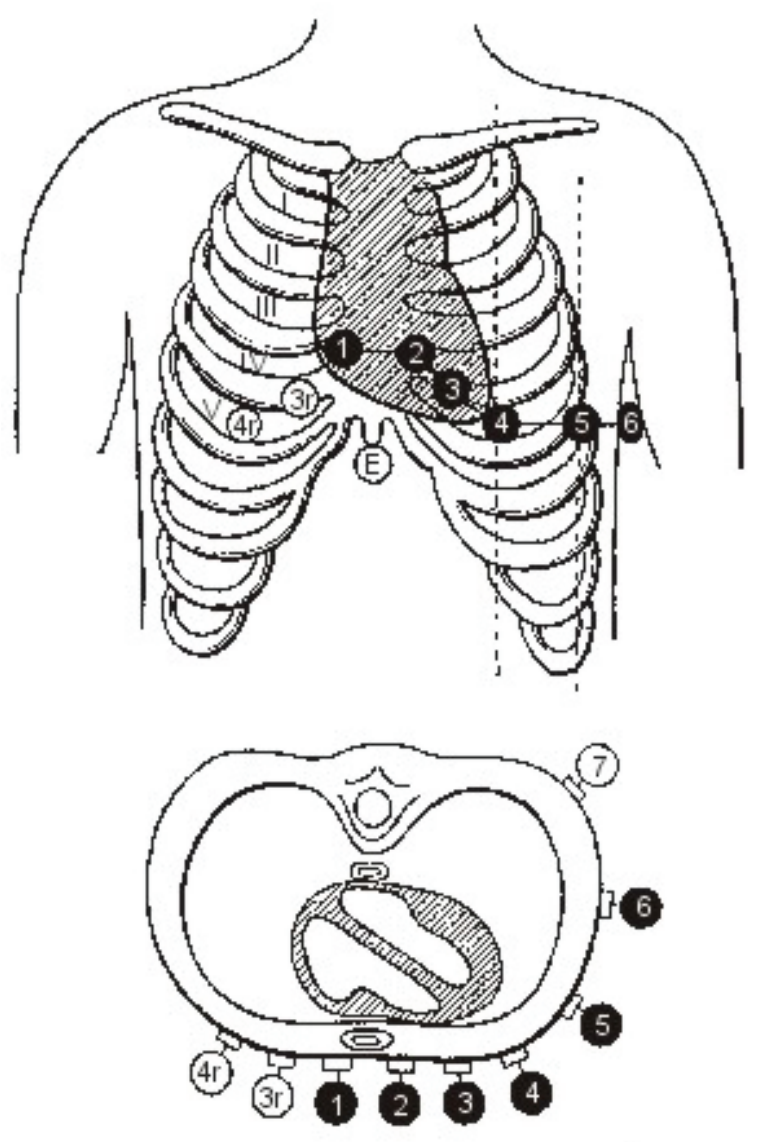

Fig. 1. Relation between a source of the electrical field and standard 12-leads system.

\section{Body surface potential mapping}

The goal of the BSPM is to determine electric activity of the heart by reconstructing maps of epicardial potentials, excitation times (isochrones), and electrograms from data measured on the body surface (Oster et al.
1998). Several types of maps were suggested in order to have better insight in the electrical heart field events (Fig. 2):

- Isopotential maps representing distribution of the electrical potential value on the torso surface in a defined moment of depolarization or repolarization processes (Fig. 2a).

- Isointegral maps representing distribution of the integrals (summarizing both amplitude and time) of the main electrical events of the cardiac cycle depolarization, repolarization or the whole electrical cycle (Fig. 2b).

- Isoareal maps representing distribution of the integrals of selected intervals of the electrical heart cycle (Fig. 2c).

- Maxima maps representing distribution of the maximal values of individual waves $\left(\mathrm{Q}, \mathrm{QS}, \mathrm{R}, \mathrm{R}^{\prime}, \mathrm{S}\right.$, T - Fig. 2d).

- Isochrone maps representing distribution of excitation time and selected intervals (Fig. 2e).

a

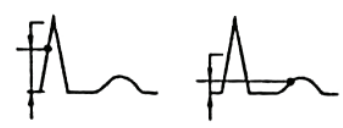

b

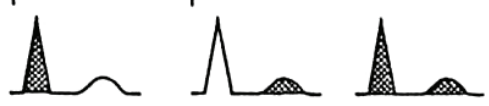

c

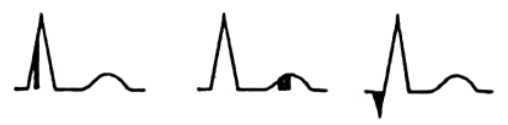

d
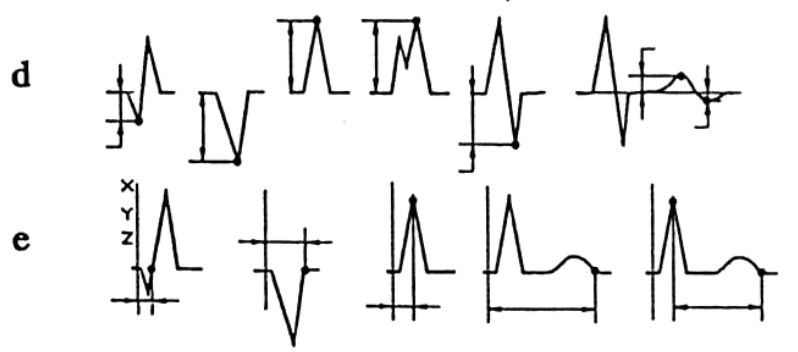

Fig. 2. Different types of body surface maps (for more details see the text).

Every mapping procedure has some specific advantages. But all procedures are limited in their ability to localize cardiac electric events because the potential at every point on the body surface is determined by the electric activity in the entire heart; moreover they are also limited in their ability to resolve multiple electric events because the volume conductor between the heart and body surfaces smoothes the potential distribution. All these facts limit a clinical use of BSPM with two exceptions where they are irreplaceable (Kittnar and Št'ovíček 1993): 
- In the arrhythmologic field the BSPM has two main objectives: 1) identification of signs of susceptibility to arrhythmias, and 2) identification of site of origin of the arrhythmias (De Ambroggi and Corlan 2007).

- In the identification of accessory pathways sites in the cases of pre-excitatory syndromes.

In any case BSPM has two major advantages over the conventional 12-lead electrocardiogram: 1) it allows exploring the entire chest surface, thus providing all the information on the cardiac electric field available at the body surface; 2) it is undoubtedly more sensitive in detecting local electrical events, such as conduction disturbances or regional heterogeneities of ventricular recovery. That is why we have used this electrocardiographic method for detailed analysis of the electrical heart field in patients suffering from coronary artery disease (Kittnar et al. 1993), in patients with metabolic risk factors of a cardiovascular mortality (Ždárská et al. 2007), with potentially cardiotoxic medication (Kittnar et al. 2004, Slavíček et al. 1998) and in people with physiologically changed geometry of thorax organs (Lechmanová et al. 2002). In addition to many other parameters of the electrical heart field we assessed particularly QT dispersion.

As myocardium is generally an inhomogeneous tissue there are regional differences in action potential duration. Therefore, one lead can represent the area of earliest repolarization by the shortest QT interval whilst another one indicates by the longest QT interval the area of the heart which is last to depolarize. The difference between them is known as QT dispersion (QTd) which is thought to be representative of the overall variability in the timing of repolarization (Day et al. 1992). However, another concept of QT dispersion origin has been discussed in the late nineties, suggesting that rather than regional heterogeneity of myocardial repolarization, different projections of the 3-dimensional T-wave loop into individual ECG leads accounted for the possible differences in QT interval duration (Kors et al. 1999, Batchvarov and Malik 2000). In order to contribute to solving the question of a clinical relevance of QTd we have used the late pregnancy as a model of the physiologically changed spatial arrangement of thoracic organs accompanied by deviated morphology of the T-loop (Lechmanová et al. 2002). Thus this model made it possible to study the relation between QT dispersion and spatial aspects of the T- loop. The results have proved increased QTd in the late pregnancy. Our results suggest that it is necessary to re-evaluate the possible clinical importance of QT dispersion. But there were three different explanations of the results:

- Changed spatial arrangement of abdominal organs during pregnancy results in increased pressure of the viscera on the diaphragm and chest organs. This is undoubtedly associated with the changed spatial position of the heart which is then accompanied by an "abnormal" pattern of the electrical heart field configuration.

- Changed electrical properties of myocardium due to changed both sympathetic and hormonal modulation (epinephrine, progesterone) of the electrical heart activity. This can be to some extent in accordance with the hypothesis of increased QT dispersion being a marker of enhanced risk of arrhythmias as similar changes of neurohumoral cardiac modulation contribute very probably to such increased risk and could be involved in delay of cardiac electrical recovery as well.

- Changed tissue impedance in tissues lying between the ventricular myocardium and measuring electrodes at the body surface due to gradual accumulation of sodium and total body water, most of which is extracellular, during pregnancy.

Thus, it can be concluded that (as usually) the results have provided more questions than answers. For solving the question if the increased QTd is caused by the rotation of the heart or by other factors a mathematical model can be used.

\section{Mathematical model as a tool for the answering the questions}

Two mathematical models (simple and anatomical) were used to test the hypothesis whether the observed changes in cardiac electrical field during late pregnancy can be attributed to the geometrical changes (rotation of the heart). Both models were used to perform a forward calculation, i.e. to calculate superficial electrograms from the dipolar source situated in the heart region. The simple model is represented by an elliptical cylinder and assumes homogenous conduction. The anatomical model is adopted from the Utah Torso model (MacLeod et al. 1991), representing detailed torso anatomy including variable organs conductivities and FEM method is used for the calculation.

The data from vectorcardiograms (VCGs) from healthy volunteers were used as a dipolar source in both models. Then, the electrical potentials on virtual torsa 


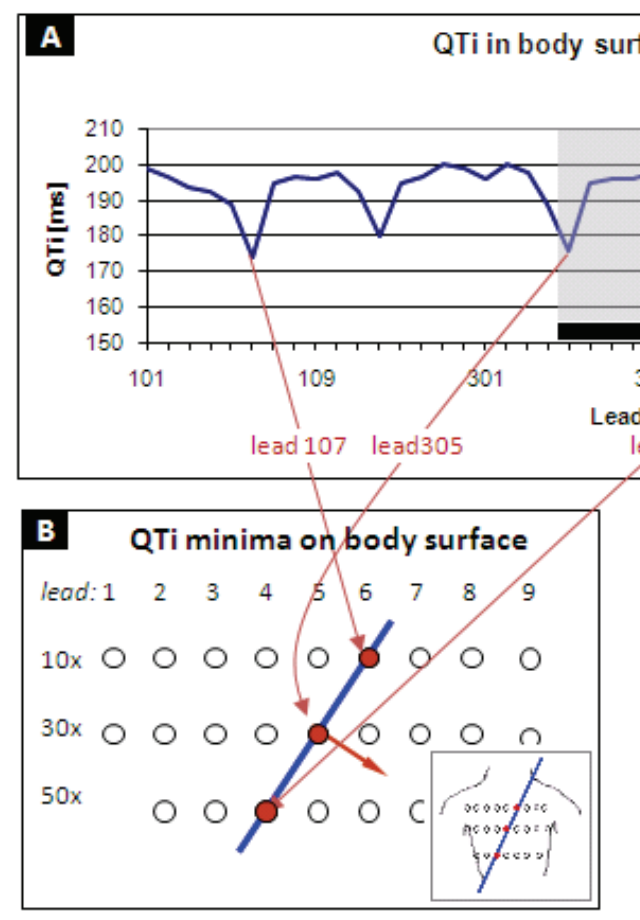

\section{QTi in body surface leads}
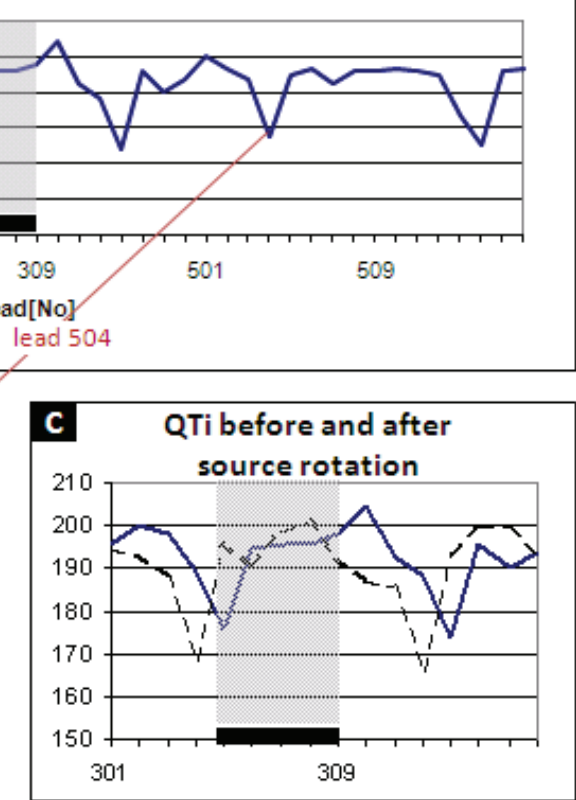

Fig. 3. Distribution of $\mathrm{QT}$ interval minima around the chest (for more details see the text). surfaces were calculated and 80 superficial electrocardiograms reconstructed. To validate the model, the calculated electrograms were compared to 80 real surface electrograms taken from respective volunteers simultaneously with VCGs. The correlation coefficients between calculated and measured superficial electrograms ranged between 0.91 and 0.98 for 20 precordial leads which was considered satisfactory. The correlation decreased to 0.8 for some of the leads further away from the heart (right chest, back) due to reasons that can include: poor quality of signals from paravertebral regions, inaccurate model representation around the upper extremities, greater effect of real torso and model differences with increasing distance from the surface.

In order to test the hypothesis, the source VCG was transformed (rotated) in the similar manner as was found during pregnancy (Lechmanová et al. 2002). Our in silico experiment (i.e. altered tissue conductivity, effects of altered hormone profiles) allowed for testing the geometry effect separate from other hypotheses above since no other parameters were changed. Then the cardiograms before and after the VCG source transformation were compared, specifically the QTd. The method of mathematical modeling brought several interesting findings:

- There was no significant difference in QTd between the original and transformed fields if analyzed from 80 -leads system. We conclude that the changes in geometry of the heart and torso are not a major mechanism responsible for alterations in cardiac electrical field.

- A detailed analysis of QT interval (QTi) revealed a periodical distribution of distinct QTi minima around the chest (Fig. 3-A). A 3D reconstruction revealed that the QTi minima are all situated in one plane (Fig. 3-B). Further analysis confirms that this plane is perpendicular to the terminal vector of repolarization (i.e. terminal T-wave electrical axis). This observation is in accord with the known presumption that no potential is recorded in the leads when these are oriented perpendicularly to the cardiac vector. For this reason, towards the end of repolarization, in some leads the $\mathrm{T}$ wave amplitude is inevitably very low or absent. These regions (sometimes referred to as zerolines) will always exhibit lowest QTi.

- For a detailed repolarization analysis (like T-wave amplitude, duration, QTi, etc.), the number of leads used is crucial. A standard 12-lead system is often insufficient to record or to display some critical aspects of cardiac field as demonstrated in Figure 3. In the graph, panel $\mathrm{C}$, the full line represents QTi duration similarly as in panel $\mathrm{A}$. The dashed line represents QTi estimated after VCG source alteration (rotation). The grey area indicates region covered by standard precordial leads V1-V6. It can be clearly seen, that in case of original data (full line), precordial leads can record QTi minimum but not its maximum. On the contrary, after the source rotation (dashed line), QTi minimum is missed while maximum is 
covered by precordial leads. Thus different durations of QTi reported from standard lead systems may be a consequence of such methodological approach to which the body surface mapping systems are essentially resistant.

- Interestingly, if comparing the results of forward calculations in simple and anatomical models, the detailed FEM torso model did not provide the results clearly superior to the simple model. It can be hypothesized, that since none of the models reflects specific torso geometry of the subjects which the VGS and BSPM data were obtained from, the calculations using both models are comparably inaccurate. This could implicate, that in order to increase accuracy of cardiac electrophysiology data interpretation and diagnostics, it is required to obtain rather accurate electro-anatomical models specific to individual patients.

\section{Conclusions}

For over a century, the cardiac field resists our detailed understanding, specifically in the areas of arrhythmogenesis and repolarization. After years of skepticism, in silico analysis is becoming a recognized method of biomedical research. We have demonstrated its usefulness in the field of cardiac electrophysiology, specifically when exploring delicate changes of ventricular repolarization. Our results imply that any attempt to interpret repolarization data needs to consider geometrical relationship between the lead system and electrical field, specifically at terminal repolarization. This includes proper selection of leads, construction of electro anatomical models specific to individual patients and identification of regions where low voltage signals result from methodology and not local pathology. Due to complexity of electrophysiology, mathematical modeling is the (only) efficient tool for such analyses.

\section{Conflict of Interest}

There is no conflict of interest.

\section{Acknowledgements}

This study is supported by grant GAAV 1ET201210527.

\section{References}

BATCHVAROV V, MALIK M: Measurement and interpretation of QT dispersion. Prog Cardiovasc Dis 42: 325-344, 2000.

DAY CP, MCCOMB JM, CAMPBELL RWF: QT dispersion in sinus beats and ventricular extrasystoles in normal hearts. Br Heart J 67: 39-41, 1992.

De AMBROGGI L, CORLAN AD: Clinical use of body surface potential mapping in cardiac arrhythmias. Anadolu Kardiyol Derg 7 (Suppl 1): 8-10, 2007.

HIGHAM PD, CAMPBELL RWF: QT dispersion. Br Heart J 71: 508-510, 1994.

KITTNAR O, PACLT I, MLČEK M, SLAVÍČEK J, DOHNALOVÁ A, HAVRÁNEK Š, BRIZMAN E, KITZLEROVÁ E, PIŠVEJCOVÁ K: QT dispersion and electrical heart field morphology in patients treated with dosulepin. Physiol Res 53: 379-386, 2004.

KITTNAR O, SLAVÍČEK J, VÁVROVÁ M, BARNA M, DOHNALOVÁ A, MÁLKOVÁ A, ASCHERMANN M, HUMHAL J, HRADEC J, KRÁL J: Repolarization Pattern of Body Surface Potential Maps (BSPM) in Coronary Artery Disease. Physiol Res 42: 123-130, 1993.

KITTNAR O, ŠŤOVÍČEK P: Contemporary Body Surface Potential Mapping in Electrocardiology and its Perspectives. Physiol Res 42: 141-143, 1993.

OSTER HS, TACCARDI B, LUX RL, ERSHLER PR, RUDY Y: Electrocardiographic imaging: Noninvasive characterization of intramural myocardial activation from inverse-reconstructed epicardial potentials and electrograms. Circulation 97: 1496-1507, 1998.

KORS JA, VAN HERPEN G, VAN BEMMEL JH: QT dispersion as an atribute of T loop morphology. Circulation 99: 1458-1463, 1999.

LECHMANOVÁ M, KITTNAR O, MLČEK M, SLAVÍČEK J, DOHNALOVÁ A, HAVRÁNEK Š, KOLAŘÍK J, PAŘÍZEK A: QT dispersion and T-loop morphology in late pregnancy and after delivery. Physiol Res 51: 121129, 2002. 
MACLEOD RS, JOHNSON CR, ERSHLER PR: Construction of an inhomogeneous model of the human torso for use in computational electrocardiography. IEEE Engineering in Medicine and Biology Society, 13th Annual International Conference. IEEE Press, 1991, pp 688-689.

SLAVÍČEK J, PACLT I, HAMPLOVÁ J, KITTNAR O, TREFNÝ Z, HORÁČEK BM: Antidepressant drugs and heart electrical field. Physiol Res 47: 297-300, 1998.

ŽDÁRSKÁ D, PELÍŠKOVÁ P, CHARVÁT J, SLAVÍČEK J, MLČEK M, MEDOVÁ E, KITTNAR O: ECG body surface mapping (BSM) in type 1 diabetic patients. Physiol Res 56: 403-410, 2007. 